\title{
Review
}

\section{Botulinum Neurotoxin Type A in the Treatment of Oily Skin and Acne: Evidence and a Proposed Mechanism}

\author{
Nark-Kyoung Rho ${ }^{1,2, *}$, Young-Chun Gil ${ }^{3}$ \\ 1 Department of Dermatology, Sungkyunkwan University School of Medicine, Seoul, South Korea; rho- \\ nark@hanmail.net \\ 2 Leaders Aesthetic Laser \& Cosmetic Surgery Center, Seoul, South Korea; rhonark@hanmail.net \\ 3 Department of Anatomy, Chungbuk National University School of Medicine, Cheongju, South Korea; gi- \\ lyc@chungbuk.ac.kr \\ * Correspondence: rhonark@hanmail.net
}

\begin{abstract}
Intradermal injection of botulinum neurotoxin is a frequently performed procedure in aesthetic dermatology to improve facial skin tone, texture, fine wrinkles, and enlarged pores. In practice, botulinum neurotoxin type $\mathrm{A}$ is also used to reduce skin oiliness of the face. There is increasing evidence that acetylcholine plays specific roles in sebum production, suggesting that botulinum neurotoxin type A may reduce sebum production by interfering with cholinergic transmission between sebaceous glands and autonomic nerve terminals. Botulinum neurotoxins can also inhibit several pathogenetic components of acne development, suggesting that botulinum neurotoxins can be used as a safe and effective treatment modality for acne and other skin disorders related to the overactivity of sebaceous glands. This review aims to explore the current evidence behind the treatment of oily skin and acne with botulinum neurotoxin type A.
\end{abstract}

Keywords: acetylcholine; acne vulgaris; botulinum toxins; cholinergic receptors; non-neuronal cholinergic system; oily skin; sebaceous glands; sebum

Key Contribution: Cholinergic receptors on the sebaceous glands are action sites of botulinum neurotoxins. Intradermal injection of botulinum neurotoxin type A can be a treatment option for treating oily skin and acne.

\section{Introduction}

A sebaceous gland (SG) is a microscopic exocrine gland in the skin that opens into a hair follicle to secrete an oily or waxy matter, called sebum. Sebum production is physiologic and serves to lubricate the hairs and the stratum corneum. However, when produced in excess, it can be an aesthetic concern for many people since it frequently accompanies greasy skin and enlargement of facial pores (Figure 1). The consequences of excess sebum may be associated with adverse psychological and social effects resulting from skin oiliness and shine, prominent pores [1]. Excessive sebum production is also a major pathogenetic factor in several dermatologic disorders such as acne [2][3]. One of the most effective inhibitors of sebum production is oral isotretinoin. However, many patients cannot tolerate, are unwilling to accept the side effects, are contraindicated for its use, or do not have severe enough disease to justify its use [2]. Studies show that lasers and other energy-based treatments can reduce sebum production. However, a shorter duration of efficacy, potential side effects, and the relative lack of evidence are common drawbacks to these treatments. 
Botulinum neurotoxin type A (BoNTA) blocks the release of acetylcholine (Ach) into the synaptic cleft where it binds to a cholinergic receptor on a post-synaptic cell. Acting at the neuromuscular junction, BoNTA leads to a loss of muscle tone, while in some glandular tissues, it inhibits cholinergic sympathetic nerve function. The ability of BoNTA to inhibit cholinergic transmission prompted further investigations into its clinical use in several autonomic disorders resulting in glandular hypersecretion, such as hyperhidrosis and sialorrhea [1][2]. Another well-known cutaneous exocrine gland is the SG, opening into a hair follicle to secrete sebum. The neuronal control over the SG has long been implicated by clinical observations [4-7]. Empirical reports on the use of BoNTA to suppress excessive sebum suggest that BoNTA could modulate the neuroendocrine control over the SGs [8]. This review summarizes in vitro and in vivo studies relevant to BoNTA therapy for SG overactivity. We also review the reported cases and clinical trials on the use of BoNTA for oily skin, wide pores, and associated acne.

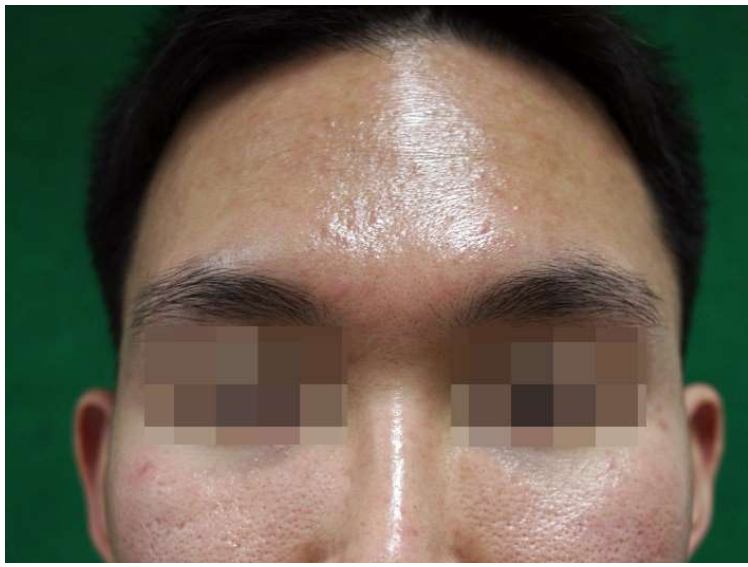

(a)

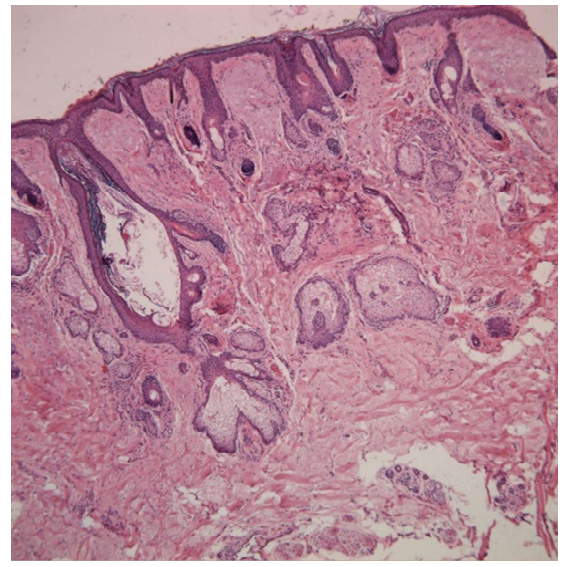

(b)

Figure 1. (a) A young Korean male with oily skin appearing greasy and shiny, accompanied by enlarged pores on the cheeks; (b) Histologic findings of the facial skin of excess sebum secretion. Note the lobules of mature sebaceous glands and a dilated follicular infundibulum filled with keratinous material (nose, hematoxylin and eosin staining, $\times 50$ ).

\section{Botulinum Neurotoxin for Sebum Reduction}

\subsection{Anecdotal Clinical Reports}

Unlike sweat secretion, sebum production has not been thought to be controlled by the autonomic nervous system [9]. It is often expressed as saying that "no nerve supply but only hormonal regulation" affects the sebaceous gland activity. However, clinical observations have suggested a neuronal control over the human SG. In patients with partial facial paralysis, variations in the sebum secretion rate were observed between the unaffected and neurologically impaired halves of the face [4]. Unilateral facial acne has also been observed following partial facial paralysis [5]. Sebum secretion from the thighs of paraplegic patients is significantly higher than that in non-paraplegic controls, while the sebum excretion rate is unchanged above the level of spinal injury [4]. Studies also showed that the use of topical anticholinergic agents resulted in a significant decrease in sebum secretion [7]. These clinical findings prompted many researchers to take a closer look at the relationship between the SG and cholinergic system, with multiple studies demonstrating the expression of ACh receptors on the SG [10][11].

\subsection{Extraneuronal Cholinergic System of Sebaceous Glands}


ACh acts through two principal receptor types, muscarinic and nicotonic [12]. The muscarinic receptor $\mathrm{m} 2 \mathrm{AChR}$ is expressed in suprabasal sebocytes, and its signaling pathways in the SGs are well-described [13-15]. In comparison, ductal cells of the SG differentially show the highest immunoreactivity for or alpha 7 nicotinic acetylcholine receptor $(\alpha 7 \mathrm{nAChR})$, a major nicotinic ACh receptor subunit [10][16][17]. The $\alpha 7 \mathrm{nAChR}$ is expressed in many non-neuronal tissues such as skin, although it was initially described as a neuronal receptor subtype [18]. The existence of non-neuronal cholinergic receptors in the SG suggests that nicotinic signaling pathways and ACh contribute to the pathophysiological processes in the pilosebaceous unit [18][19]. In both cultured sebocytes and healthy volunteers, ACh dose-dependently increased the lipid synthesis (Figure 2) by its interaction with $\alpha 7 \mathrm{nAChR}$ via activation of ERK signaling [19], probably through promoting sebocyte differentiation [20]. It aligns with an immunohistochemical finding that mature sebocytes, in particular, show high positivity for $\alpha 7 \mathrm{nAChR}$ [10]. In vitro, sebocytes from oily skin are phenotypically fully mature and express more cholinergic receptors [19]. These findings suggest that ACh and its receptors play specific roles in sebocyte differentiation and sebum production [10][19][20], and it is plausible to assume that oily skin may be more susceptible to cholinergic regulation than normal skin [19][21]. It remains to be seen how the human SG in vivo responds to the cholinergic stimuli in vivo. It has not been elucidated whether high doses of or prolonged exposure to Ach leads to depolarizing and desensitizing block of cholinergic receptor neurotransmission.

\section{Control}

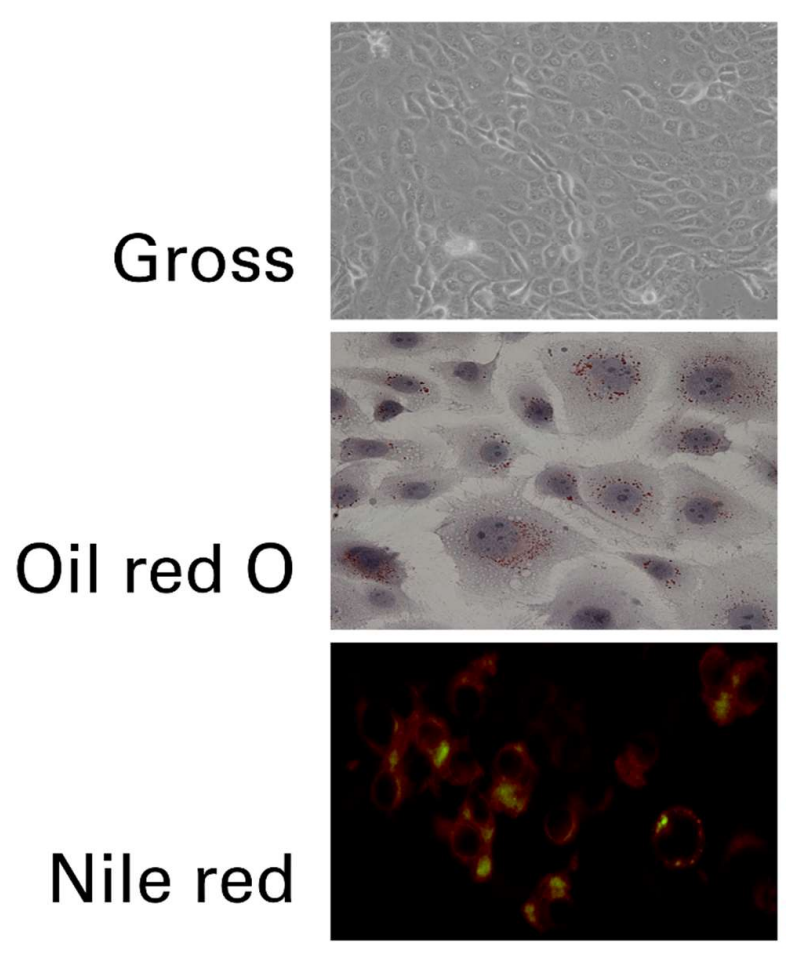

Ach 10nM
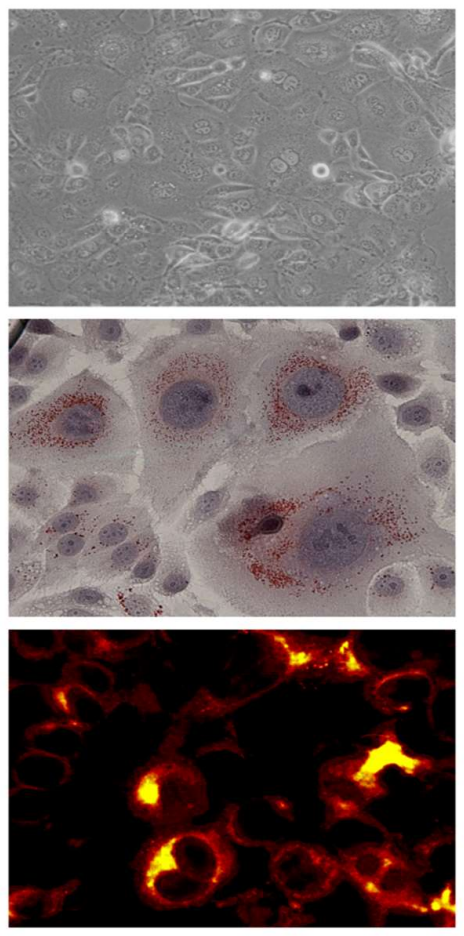

Figure 2. Increased lipid synthesis in cultured sebocytes after treatment with $10 \mathrm{nM}$ of acetylcholine. Intracellular lipids are detected in acetylcholine-treated sebocytes by lipid staining (Photos are used with kind permission of Dr. Myung Im, MD, IM Dermatology Clinic, Daejeon, Korea).

\subsection{Early Observations}


More than 40 years ago, researchers found that anticholinergics block cholinergic signaling and suppress ACh-induced sebum synthesis [19][21]. In a clinical trial involving ten patients with mild acne, a long-term application of a topical poldine methylmethosulphate, an anticholinergic agent, to the forehead skin produced a significant reduction in sebum excretion [7]. A marked decrease in sebum production and pore size was noted after oily skin treatment with $\alpha$-bungarotoxin, a well-known antagonist of the ACh receptor [18][22]. So, it is not strange to expect that BoNTA, a potent inhibitor of ACh release from cholinergic nerves, may have a suppressive effect on the SG. An animal study demonstrated a reduction in the number of sebocytes after BoNTA injection [23]. Clinically, anecdotal reports describe a decrease of facial sebum secretion after injection of BoNTA. Regression of the sebaceous cyst was described after BoNTA injection for the treatment of migraine [24]. Jankovic [25] suggested that facial seborrhea in Parkinson's disease may respond to botulinum neurotoxins. The prevalence of local skin dryness, suggestive of reduced sebum secretion, was $8 \%$ in one clinical study to evaluate the safety and efficacy of BoNTA injection to treat forehead wrinkles [26]. Wu [27] described a decreased sebum production after intradermal injection of BoNTA for cosmetic purposes. These laboratory findings and clinical observations raised speculation that BoNTA may be an effective treatment for excessive sebum production, which prompted further clinical trials.

\subsection{OnabotulinumtoxinA}

A report by Shah [28] was one of the early clinical trials that demonstrated a favorable result of BoNTA in reducing sebum production and pore size. In this retrospective study, twenty subjects with oily skin and enlarged pores were treated using an intradermal injection of onabotulinumtoxinA (Botox; Allergan, California, USA) to the "T-zone" of the face and photographically evaluated one month after treatment. Seventeen of 20 subjects showed improvement in oiliness after one treatment, with no significant complications. This study has been quoted as the first step towards a more profound understanding of BoNTA actions on the sebaceous glands. However, the lack of objective measurements strongly limits the study since the author used a photographic assessment to measure sebum production and pore size, as criticized by other researchers that the decrease in oiliness and shininess noted in the photograph may represent a decrease in sweating [2]. This study addresses the need for objective evaluation to quantitatively assess the decrease of sebum production and exclude confounding factors.

Sebumeter is a device that quantifies the sebum amount from the skin in an objective manner. A photoelectric receiver measures the amount of light passing through the sebum-covered translucent element and calculates the result [29]. Several researchers used this device to objectively measure the sebum production before and after BoNTA injections (Table 1). In a study involving 20 patients with oily skin, the researchers used a sebumeter to measure the change of sebum production after intradermal injection of onabotulinumtoxinA [30]. There was an average decrease in sebum production of $36.2 \%$ at two weeks, $29.9 \%$ at four weeks, and $25.6 \%$ at six weeks follow-up with patients' satisfaction up to 3 months. In another clinical trial of 15 females who had been injected BoNTA in glabella and forehead muscles, sebumeter readings showed a maximum decrease in sebum production $(26.8 \%)$ four weeks after treatment and returned to baseline after 16 weeks. Another group of investigators reported a $24.3 \%$ decrease in skin surface lipid at two weeks after intradermal injection of BoNTA in 10 patients with acne [31], similar to previous studies.

Subsequent investigations came with a better study design. Li and colleagues [19] performed a double-blind, placebo-controlled, split-face study and investigated the effects of BoNTA on sebum production in 20 volunteers. One side of the face was injected with BoNTA and the other with normal saline. There was a significant decrease in sebum production in the group with oily skin on the BoNTA-treated side compared to the salineinjected side, particularly at four weeks after injection. Interestingly, there was no 
significant difference between the skin on both sides of the face in the group with dry-tonormal skin. If so, it is plausible to think that oily skin may be more susceptible to cholinergic regulation than normal or dry skin.

Min et al. [32] investigated the dose-response relationship of BoNTA and sebum secretion. In their prospective, randomized, double-blind, dose-comparative clinical trial, 42 female volunteers with forehead rhytides have randomly received an injection of either low dose (10 units) or high dose (20 units) of onabotulinumtoxinA, administered in five standard injections. The sebumeter results illustrated that BoNTA injection significantly reduced sebum excretion at the injection site, with a sebum production gradient surrounding the injection point. Surprisingly, the higher injection did not significantly improve the efficacy of BoNTA.

Table 1. Quantitative studies on the effect botulinum neurotoxins on sebum secretion in patients with oily skin

\begin{tabular}{|c|c|c|c|c|}
\hline First author & Product & Injection & $\mathbf{n}$ & Findings (as described in the paper) \\
\hline Min [32] & ONA & $\mathrm{IM}$ & 42 & $\begin{array}{l}\text { A significant decrease in sebum production } \\
\text { A sebum gradient surrounding the injection point } \\
\text { No difference in the efficacy between the } 10 \text { units group } \\
\text { and the } 20 \text { units group } \\
\text { Recovery of the sebum production at the } 16 \text {-week } \\
\text { follow-up }\end{array}$ \\
\hline Kondrateva [43] & ONA & $\mathrm{IM}$ & 15 & $\begin{array}{l}\text { Maximum decrease in sebum production at the } 4 \text {-week } \\
\text { follow-up } \\
\text { A return-to-baseline at } 16 \text { weeks after injection }\end{array}$ \\
\hline Hathout [30] & ONA & ID & 20 & $\begin{array}{l}\text { A significant decrease in sebum production } \\
\text { Patient satisfaction up to } 3 \text { months } \\
\text { Associated improvement in the skin tone and facial } \\
\text { pores } \\
\text { No influence of age, gender, or skin types }\end{array}$ \\
\hline Shirshakova [31] & ONA & ID & 12 & $\begin{array}{l}\text { A decrease of skin surface sebum amount at } 1 \text { week and } \\
2 \text { weeks after injection }\end{array}$ \\
\hline $\operatorname{Li}[19]$ & MTX & ID & 20 & $\begin{array}{l}\text { A marked decrease in sebum production on the BoNTA- } \\
\text { treated side in oily skin group }\end{array}$ \\
\hline Rose [2] & $\mathrm{ABO}$ & ID & 25 & $\begin{array}{l}\text { A significantly lower sebum production at } 1,4,8 \text {, and } 12 \\
\text { weeks after injection }\end{array}$ \\
\hline Kesty [34] & $\mathrm{ABO}$ & ID & 50 & $\begin{array}{l}\text { A significant decrease in sebum production in the } \\
\text { treatment groups } 30 \text { units and } 45 \text { units } \\
\text { The effect lasted for } 6 \text { months after injection }\end{array}$ \\
\hline
\end{tabular}

ABO: abobotulinumtoxinA (Dysport; Ipsen, Wrexham, UK); ID: intradermal injection; IM: intramuscular injection; MTX: Meditoxin (Medy-Tox Inc., Seoul, Korea); ONA: onabotulinumtoxinA (Botox; Allergan, California, USA)

\subsection{IncobotulinumtoxinA and AbobotulinumtoxinA}

A recent study by Park et al. [33] included 20 patients treated with incobotulinumtoxinA (Xeomin; Merz Pharmaceuticals, Frankfurt, Germany) to improve facial skin laxity, sebum secretion, and facial pores. Sebum secretion decreased at one week, and the results were sustained through 12 weeks. All outcomes showed maximum improvement after four weeks. Their findings suggest that not only onabotulinumtoxinA but also incobotulinumtoxinA inhibits sebum production in oily skin. Differences in clinical performance 
make different BoNTA products not interchangeable. Product comparison between onabotulinumtoxinA and abobotulinumtoxinA (Dysport; Ipsen, Wrexham, UK) is welldescribed elsewhere. In the literature, we found two clinical trials on the efficacy of abobotulinumtoxinA to reduce sebum production. In their prospective, non-controlled clinical trial, Rose and Goldberg [2] injected abobotulinumtoxinA into the forehead of 25 subjects with oily skin. Intradermal injection of abobotulinumtoxinA resulted in a significant reduction in sebum production at every follow-up point (Figure 3). An average decrease in the sebumeter reading was $80 \%$ at a 1-month follow-up, comparable with the sebum reduction rate after oral isotretinoin. In a randomized, double-blinded, placebocontrolled study by Kesty and Goldberg [34], subjects who received 30 or 45 units of abobotulinumtoxinA showed statistically significant decreases of sebumeter readings compared to both the untreated group and the subjects treated with 15 units. Sebum reduction lasted for six months, which is much longer than expected. These results encourage the question of whether abobotulinumtoxinA is more effective than onabotulinumtoxinA in treating oily skin.

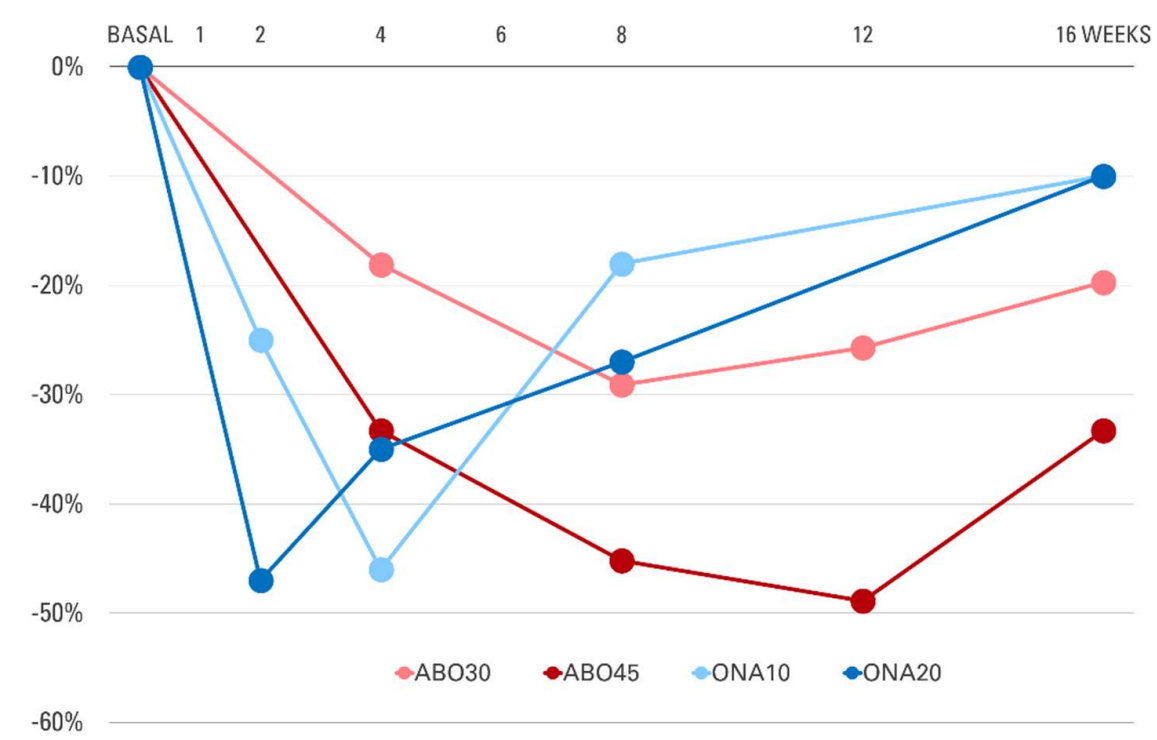

Figure 3. Changes of sebum secretion relative to baseline at each follow-up point after injection of botulinum toxin type A into the forehead. ABO30: abobotulinumtoxinA 30 units; ABO45: abobotulinumtoxinA 45 units; ONA10: onabotulinumtoxinA 10 units; ONA20: onabotulinumtoxinA 20 units. Data from [32][34].

\subsection{Enlarged Facial Pores}

Besides a decrease in the sebum secretion, the BoNTA-treated subjects frequently report the associated improvement in the general texture of the skin, highlighted by the "pores shrinkage" [30]. According to Wu [27], the intradermal injection of BoNTA results in an improvement in a decrease in sebum production and a reduction in the number of prominent facial pores. The finding was reaffirmed by Liew [35], demonstrating a reduction of skin pore size at six weeks following intradermal injection of BoNTA in the midface. Considering the sebum output level correlates most significantly with facial pore size [2][36], it is not surprising that BoNTA injection reduces not only the sebum production but also the pore size, as demonstrated in multiple clinical studies [2][19][37][38]. Since nicotinic Ach receptors influence the tissue remodeling activity of dermal fibroblasts [39], a blockade of cholinergic signals by BoNTA may further help diminish prominent facial pores. There are clinical investigations that show the pore improvement efficacy of BoNTA using objective measurements [33][40][41]. However, other factors, such as 
epidermal regeneration and neocollagenesis after multiple needle pricks, might have contributed to the pore improvement [42].

\subsection{Enlarged Facial Pores}

Besides a decrease in the sebum secretion, the BoNTA-treated subjects frequently report the associated improvement in the general texture of the skin, highlighted by the "pores shrinkage" [30]. According to Wu [27], the intradermal injection of BoNTA results in an improvement in a decrease in sebum production and a reduction in the number of prominent facial pores. The finding was reaffirmed by Liew [35], demonstrating a reduction of skin pore size at six weeks following intradermal injection of BoNTA in the midface. Considering the sebum output level correlates most significantly with facial pore size [2][36], it is not surprising that BoNTA injection reduces not only the sebum production but also the pore size, as demonstrated in multiple clinical studies [2][19][37][38]. Since nicotinic Ach receptors influence the tissue remodeling activity of dermal fibroblasts [39], a blockade of cholinergic signals by BoNTA may further help diminish prominent facial pores. There are clinical investigations that show the pore improvement efficacy of BoNTA using objective measurements [33][40][41]. However, other factors, such as epidermal regeneration and neocollagenesis after multiple needle pricks, might have contributed to the pore improvement [42].

\subsection{Discomfort and Side Effects}

Most clinical trials presented in this review describe an intradermal injection technique, and in only two studies [32][43], the injection depth was intramuscular. Intramuscular injection is generally not recommended when treating oily skin with BoNTA because it may influence the underlying facial expression muscles [2]. Apart from the pain during intradermal injection, BoNTA treatment of oily skin has been reported to be generally well-tolerated by the patient and associated with no significant adverse effects such as allergic reaction, facial palsy, or severe paralysis of muscles adjacent to the site of injection. Some patients reported a mild-to-moderate stinging sensation on the injection site, which was temporary and self-limiting [19]. A decrease of the frontalis muscle tension can occur when BoNTA is injected into the inferior parts of the forehead [2]. A bruise can occur, but according to the authors' experience, the risk of bruising can be minimized by keeping the injection depth strictly intradermal.

\section{Botulinum Neurotoxin for Acne Treatment: Possible Mechanisms}

\subsection{Pathogenesis of Acne}

Acne vulgaris is a multifactorial chronic inflammatory disorder of the pilosebaceous unit [44]. Four major factors are known to be involved in the pathogenesis of acne: (1) increased sebum production with altered lipid composition, (2) metagenomic modifications of the bacterial microbiome leading to the Cutibacterium acnes (formerly Propionibacterium acnes) biofilm formation, (3) abnormal keratinization of the infundibulum and the resulting comedogenesis, and (4) inflammatory cytokine secretion and the infiltration of inflammatory cells into the perifollicular dermis [44][45]. While all four factors are interdependent, it is generally accepted that alterations of the SG and the sebum represent the initial event influencing other pathological processes of acne development. In an acne subject, sebaceous glands and the entire sebaceous follicle are much larger than in non-acne subjects [46]. An early, non-inflammatory acne lesion (a "comedone") develops when the infundibulum is filled with excessive sebum, and the pore is plugged by the sloughed follicular keratinocytes [45][47]. Considering its inhibitory action on the SG, BoNTA can be a therapeutic modality for treating acne with excessive sebum production.

\subsection{Acne and Cholinergic Signaling}


A growing number of scientific findings indicate that acne may result from an altered cholinergic response of the pilosebaceous unit [44][48]. Several lines of clinical evidence suggest that an exacerbation of acne is related to activation of the stress response through cholinergic signaling [19]. One good example is a positive correlation between cigarette smoking and acne [49][50]. Patients with long-term exposure to nicotine, a major component of tobacco, frequently show an increased sebum secretion, which may cause an acne exacerbation [19]. Human SGs strongly express nAChRa7, meaning that their glandular function is under the regulation of cholinergic signals, at least in part [10][18-21].

Not only the SG but also the follicular epithelium is under the control of the cholinergic system. There is ample evidence that $\mathrm{ACh}$ and nicotine play essential roles in keratinocyte adhesion, migration, differentiation, and apoptosis [12][51] and may participate in acne pathogenesis by promoting infundibular epithelial hyperplasia and thus follicular plugging [19]. Data show that nicotine and the non-neuronal cholinergic system play a causative role in the pathogenesis of hidradenitis suppurativa, a chronic inflammatory disorder of the pilosebaceous unit, by stimulating the sebum secretion and the proliferation of the follicular epithelium [48]. By blocking the cholinergic signaling, BoNTA may increase the motility of the infundibular keratinocytes, which enables better clearance of hair follicle opening and prevents the formation of comedones.

Several clinical observations imply the role of BoNTA in the treatment of acne. A neurologic report describes a marked improvement of facial acne following BoNTA injections to treat facial tics in patients with Tourette syndrome [52]. In the report, clearing of perinasal acne was observed 1-2 weeks after injecting 20 to 25 units of onabotulinumtoxinA into the paranasal facial expression muscles. The clinical improvement lasted about four months. In a controlled study of BoNTA for the treatment of facial rhytides, acne developed at a lower rate in the treatment group than in the placebo group [53]. Intradermal injection of BoNTA is particularly effective for treating acne on the forehead, associated with excess sebum secretion (Figure 4).
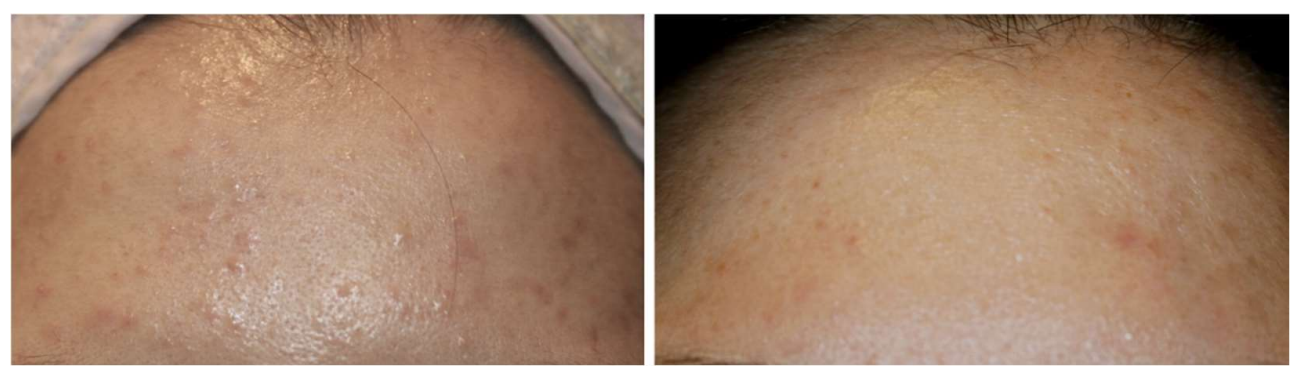

Figure 4. A young Korean female with oily skin and acne on the forehead. Two weeks after intradermal injection of 12 units of onabotulinumtoxinA, a significant improvement is observed.

\subsection{Catecholamines}

Changes in acne severity correlate highly with increasing emotional stress [54]. The most widely accepted explanation may be the role of adrenergic signaling in the functional regulation of SGs [55]. Upon a stress response, our body triggers the sympathetic-adrenomedullary axis to release catecholamines (epinephrine, norepinephrine, and dopamine), to which human SGs respond [55][56]. Sebocytes treated with norepinephrine or epinephrine showed an increase in intracellular lipid accumulation in a dose-dependent manner. Norepinephrine and epinephrine in sebocytes augment the transcription of the sebaceous gland differentiation markers [57]. Also, catecholamines increase biofilm formation and stimulate sebocyte lipid synthesis by C. acnes [58]. Botulinum neurotoxins modulate sympathetic neurons during intense activation by physiological or pathophysiological stimuli, as shown by Zhou et al. [59] that BoNTA inhibits adrenergic response in a dose-dependent manner by cleaving SNAP-25 in sympathetic neurons. Data from an animal study showed that BoNTA induces prolonged sympathetic block when placed on sympathetic ganglia 
[60]. A clinical trial in patients with complex regional pain syndrome also demonstrated a sympathetic inhibition by BoNTA [61]. Several studies [62-64] highly suggest an inhibitory effect of botulinum neurotoxins on the secretion of catecholamines, providing further rationale for the use of BoNTA in acne treatment. The detailed mechanism of BoNTA affecting the sympathetic pathway needs to be further investigated.

\subsection{Mediators of Inflammation in Acne}

A frequent exacerbation of acne during periods of emotional stress is also related to neuropeptide secretion and the resulting inflammation. Among many neurogenic peptides, substance $P$ is well known to contribute to the onset and the exacerbation of inflammation in acne. In vitro study results suggest that substance P stimulates the lipogenesis in the SGs of acne patients, followed by the proliferation of $C$. acnes and the provocation of inflammatory reactions [65]. Knowing that BoNTA prevents the release of substance P both in vitro and in vivo [66], its anti-inflammatory properties may help resolve inflammation in acne and other disorders of the hair follicle [67]. Findings from a series of investigations [68][69] also suggest that BoNTA may benefit acne patients by blocking ACh and inhibiting the release of several neurogenic transmitters.

When $C$. acnes and other bacterial species act on the SG lipid to stimulate the release of cytokines, sebaceous follicles release various inflammatory mediators. Arachidonic acid is one of the crucial inflammatory mediators in acne pathogenesis and exerts diverse roles in the development of acne inflammation [70][71]. BoNTA exerts an anti-inflammatory role in acne by lowering the intraterminal arachidonic acid level [72]. Interestingly, arachidonic acid is known to induce a release of ACh at cholinergic nerve terminals [72], which BoNTA can best suppress. In an animal study [73], BoNTA also inhibited the expression of cyclooxygenase-2, a key enzyme for cytokine-mediated acne inflammation [70]. Inflammatory acne lesions show an increased number and activity of mast cells in their SGs [65][74]. BoNTA inhibits mast cell activity by affecting soluble N-ethylmaleimide-sensitive-factor attachment protein receptor (SNARE) proteins, including synaptosomal-associated protein-25 (SNAP-25) and vesicle-associated membrane protein (VAMP) [75]. BoNTA also inhibits signaling of the transient receptor potential vanilloid subtype 1 (TRPV1) [76], which is a regulator of human sebocyte activities and the disease process of acne [77][78]. Of note, TRPV1 is an important pathogenetic factor in inflammatory process of pain syndromes, chronic itch, and rosacea, all known indications of BoNTA.

Collectively, all these findings suggest that a strong involvement of inflammatory mediators in the SG can be a target of acne treatment using BoNTA.

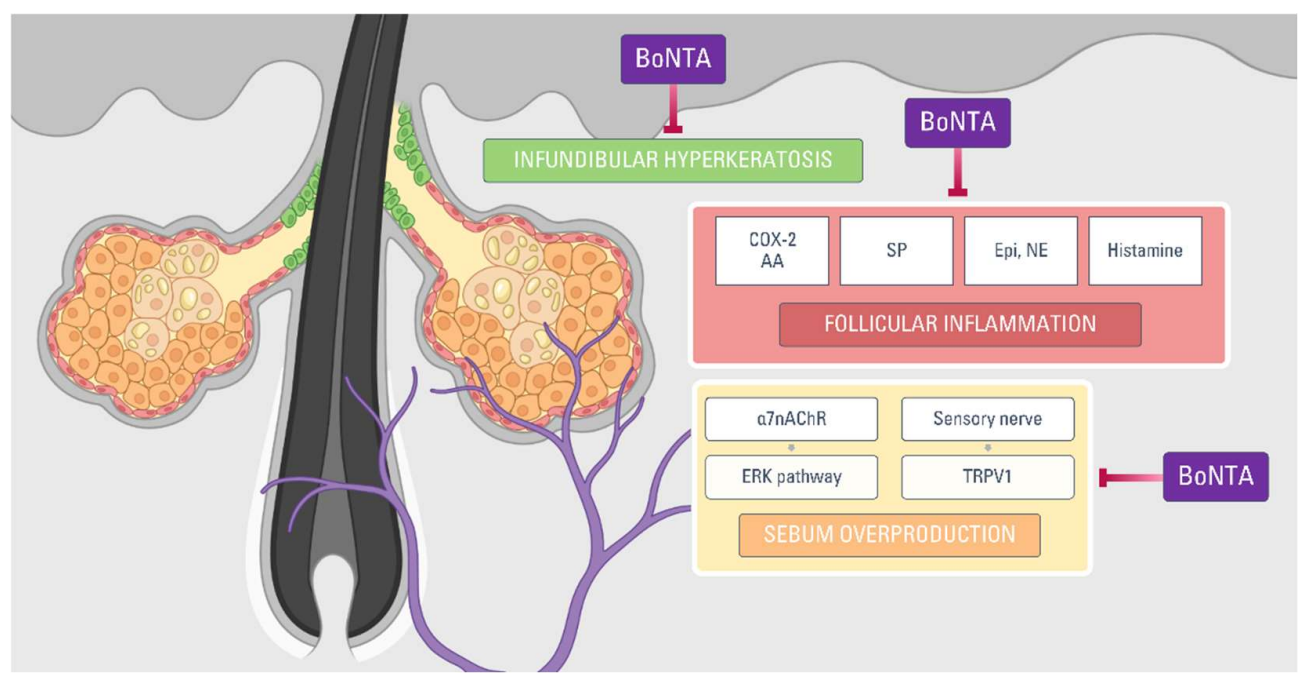


Figure 5. Pathogenetic factors of acne that can be inhibited by botulinum neurotoxin type A. $\alpha 7 \mathrm{nA}$ ChR: alpha 7 nicotinic acetylcholine receptor; AA: arachidonic acid; BoNTA: botulinum neurotoxin type A; COX-2: cyclooxygenase-2; Epi: epinephrine; NE: norepinephrine; SP: substance P; TRPV1: transient receptor potential vanilloid subtype 1 .

\section{Conclusions}

BoNTA affects cholinergic transmission and other neurotransmitter pathways of SG activities. Data from in vitro and in vivo studies indicate that BoNTA can be a potential tool to treat oily skin and acne, closely related to abnormal sebaceous gland activities (Figure 5). A review of clinical trials revealed that intradermal injection of BoNTA is an effective and safe treatment of excessive sebum secretion and prominent facial pores. Since BoNTA is not labeled for acne treatment, it should be kept as a secondary option in acne management until more clinical trials confirm these promising results. Further research is recommended to present more robust evidence for a functional role of BoNTA in SG biology.

Conflicts of Interest: The authors declare no conflict of interest. 


\section{References}

1. Arbuckle, R.; Atkinson, M.J.; Clark, M.; Abetz, L.; Lohs, J.; Kuhagen, I.; Harness, J.; Draelos, Z.; Thiboutot, D.; Blume-Peytavi, U.; et al. Patient experiences with oily skin: The qualitative development of content for two new patient reported outcome questionnaires. Health Qual. Life Outcomes 2008, 6, 1-15, doi:10.1186/1477-7525-6-80.

2. Rose, A.E.; Goldberg, D.J. Safety and efficacy of intradermal injection of botulinum toxin for the treatment of oily skin. Dermatol. Surg. 2013, 39, 443-448, doi:10.1111/dsu.12097.

3. Endly, D.C.; Miller, R.A. Oily skin: a review of treatment options. J. Clin. Aesthet. Dermatol. 2017, 10, 49-55.

4. Burton, J.L.; Cunliffe, W.J.; Saunders, I.G.G.; Shuster, S. The effect of facial nerve paresis on sebum excretion. Br. J. Dermatol. 1971, 84, 135-138, doi:10.1111/j.1365-2133.1971.tb06856.x.

5. Sudy, E.; Urbina, F. Unilateral acne after facial palsy. An. Bras. Dermatol. 2018, 93, 441-442, doi:10.1590/abd1806-4841.20187437.

6. Thomas, S.E.; Conway, J.; Ebling, F.J.G.; Harrington, C.I. Measurement of sebum excretion rate and skin temperature above and below the neurological lesion in paraplegic patients. Br. J. Dermatol. 1985, 112, 569-573, doi:10.1111/j.1365-2133.1985.tb15265.x.

7. Cartlidge, M.; Burton, J.L.; Shuster, S. The effect of prolonged topical application of an anticholinergic agent on the sebaceous glands. Br. J. Dermatol. 1972, 86, 61-63, doi:10.1111/j.1365-2133.1972.tb01892.x.

8. Shuo, L.; Ting, Y.; KeLun, W.; Rui, Z.; Rui, Z.; Hang, W. Efficacy and possible mechanisms of botulinum toxin treatment of oily skin. J. Cosmet. Dermatol. 2019, 18, 451-457, doi:10.1111/jocd.12866.

9. Martignoni, E.; Godi, L.; Pacchetti, C.; Berardesca, E.; Vignoli, G.P.; Albani, G.; Mancini, F.; Nappi, G. Is seborrhea a sign of autonomic impairment in Parkinson's disease? J. Neural Transm. 1997, 104, 1295-1304, doi:10.1007/BF01294730.

10. Kurzen, H.; Berger, H.; Jäger, C.; Hartschuh, W.; Näher, H.; Gratchev, A.; Goerdt, S.; Deichmann, M. Phenotypical and molecular profiling of the extraneuronal cholinergic system of the skin. J. Invest. Dermatol. 2004, 123, 937-949, doi:10.1111/j.0022202X.2004.23425.x.

11. Kurzen, H.; Wessler, I.; Kirkpatrick, C.J.; Kawashima, K.; Grando, S.A. The non-neuronal cholinergic system of human skin. Horm. Metab. Res. 2007, 39, 125-135, doi:10.1055/s-2007-961816.

12. Grando, S.A.; Zelickson, B.D.; Kist, D.A.; Weinshenker, D.; Bigliardi, P.L.; Wendelschafer-Crabb, G.; Kennedy, W.R.; Dahl, M. V. Keratinocyte muscarinic acetylcholine receptors: Immunolocalization and partial characterization. J. Invest. Dermatol. 1995, 104, 95-100, doi:10.1111/1523-1747.ep12613582.

13. Zouboulis, C.C. The brain of the skin: sebaceous gland. In Lipids and Skin Health; Pappas, A., Ed.; Springer: New York, 2015; pp. 109-125 ISBN 9783319099439.

14. Makrantonaki, E.; Adjaye, J.; Herwig, R.; Brink, T.C.; Groth, D.; Hultschig, C.; Lehrach, H.; Zouboulis, C.C. Age-specific hormonal decline is accompanied by transcriptional changes in human sebocytes in vitro. Aging Cell 2006, 5, 331-344, doi:10.1111/j.1474-9726.2006.00223.x.

15. Makrantonaki, E.; Brink, T.C.; Zampeli, V.; Elewa, R.M.; Mlody, B.; Hossini, A.M.; Hermes, B.; Krause, U.; Knolle, J.; Abdallah, M.; et al. Identification of biomarkers of human skin ageing in both genders. Wnt Signalling - A label of skin ageing? PLoS One 2012, 7, 1-10, doi:10.1371/journal.pone.0050393.

16. Ramot, Y.; Böhm, M.; Paus, R. Translational neuroendocrinology of human skin: Concepts and perspectives. Trends Mol. Med. 2021, 27, 60-74, doi:10.1016/j.molmed.2020.09.002.

17. Zouboulis, C.C.; Baron, J.M.; Böhm, M.; Kippenberger, S.; Kurzen, H.; Reichrath, J.; Thielitz, A. Frontiers in sebaceous gland biology and pathology. Exp. Dermatol. 2008, 17, 542-551, doi:10.1111/j.1600-0625.2008.00725.x.

18. Stegemann, A.; Böhm, M. Targeting the $\alpha 7$ nicotinic acetylcholine receptor - A novel road towards the future treatment of skin diseases. Exp. Dermatol. 2020, 29, 924-931, doi:10.1111/exd.14173.

19. Li, Z.J.; Park, S.B.; Sohn, K.C.; Lee, Y.; Seo, Y.J.; Kim, C.D.; Kim, Y.S.; Lee, J.H.; Im, M. Regulation of lipid production by acetylcholine signalling in human sebaceous glands. J. Dermatol. Sci. 2013, 72, 116-122, doi:10.1016/j.jdermsci.2013.06.009.

20. Kurzen, H.; Schallreuter, K.U. Novel aspects in cutaneous biology of acetylcholine synthesis and acetylcholine receptors. Exp. Dermatol. 2004, 13, 27-30, doi:10.1111/j.1600-0625.2004.00258.x.

21. Valente Duarte De Sousa, I.C. Novel pharmacological approaches for the treatment of acne vulgaris. Expert Opin. Investig. Drugs 2014, 23, 1389-1410, doi:10.1517/13543784.2014.923401.

22. Shi, V.Y.; Leo, M.; Hassoun, L.; Chahal, D.S.; Maibach, H.I.; Sivamani, R.K. Role of sebaceous glands in inflammatory dermatoses. J. Am. Acad. Dermatol. 2015, 73, 856-863, doi:10.1016/j.jaad.2015.08.015.

23. Kucukkaya, D.; Irkoren, S.; Ozkan, S.; Sivrioglu, N. The effects of botulinum toxin A on the wound and skin graft contraction. J. Craniofac. Surg. 2014, 25, 1908-1911, doi:10.1097/SCS.0000000000000941.

24. Turner, I.M.; Agrillo, T. Migraine, botulinum toxin type-A, and the disappearing sebaceous cyst. Headache 2005, 45, 166-167, doi:10.1111/j.1526-4610.2005.05034_1.x.

25. Jankovic, J. Disease-oriented approach to botulinum toxin use. Toxicon 2009, 54, 614-623, doi:10.1016/j.toxicon.2008.11.013.

26. Bulstrode, N.W.; Grobbelaar, A.O. Long-term prospective follow-up of botulinum toxin treatment for facial rhytides. Aesthet. Plast. Surg. 2002, 26, 356-359, doi:10.1007/s00266-002-2047-1. 
27. Wu, W.T.L. Microbotox of the lower face and neck: Evolution of a personal technique and its clinical effects. Plast. Reconstr. Surg. 2015, 136, 92S-100S, doi:10.1097/PRS.0000000000001827.

28. Shah, A.R. Use of intradermal botulinum toxin to reduce sebum production and facial pore size. J. Drugs Dermatol. 2008, 7, 847-850.

29. Pande, S.Y.; Misri, R. Sebumeter. Indian J. Dermatol. Venereol. Leprol. 2005, 71, 444-446, doi:10.4103/0378-6323.18959.

30. Hathout, H.M. Intradermal Injection of Botulinum Toxin A in Oily Skin, Ein Shams University, 2014.

31. Shirshakova, M.; Morozova, E.; Sokolova, D.; Pervykh, S.; Smirnova, L. The effectiveness of botulinum toxin type A (BTX-A) in the treatment of facial skin oily seborrhea, enlarged pores, and symptom complex of post-acne. Int. J. Dermatol. 2021, 1-10, doi:10.1111/ijd.15574.

32. Min, P.; Xi, W.; Grassetti, L.; Trisliana Perdanasari, A.; Torresetti, M.; Feng, S.; Su, W.; Pu, Z.; Zhang, Y.; Han, S.; et al. Sebum production alteration after botulinum toxin type a injections for the treatment of forehead rhytides: A prospective randomized double-blind dose-comparative clinical investigation. Aesthet. Surg. J. 2015, 35, 600-610, doi:10.1093/asj/sju150.

33. Park, J.-Y.; Cho, S.I.; Hur, K.; Lee, D.H. Intradermal microdroplet injection of diluted incobotulinumtoxin-A for sebum control, face lifting, and pore size improvement. J. Drugs Dermatol. 2021, 20, 49-54, doi:10.36849/JDD.2021.5616.

34. Kesty, K.; Goldberg, D.J. A randomized, double-blinded study evaluating the safety and efficacy of abobotulinumtoxinA injections for oily skin of the forehead: A dose-response analysis. Dermatol. Surg. 2021, 47, 56-60.

35. Liew, S. Discussion: Microbotox of the lower face and neck: Evolution of a personal technique and its clinical effects. Plast. Reconstr. Surg. 2015, 136, $101 \mathrm{~S}$ - 103, doi:10.1097/PRS.0000000000001840.

36. Roh, M.; Han, M.; Kim, D.; Chung, K. Sebum output as a factor contributing to the size of facial pores. Br. J. Dermatol. 2006, 155, 890-894.

37. Sapra, P.; Demay, S.; Sapra, S.; Khanna, J.; Mraud, K.; Bonadonna, J. A single-blind, split-face, randomized, pilot study comparing the effects of intradermal and intramuscular injection of two commercially available botulinum toxin A formulas to reduce signs of facial aging. J. Clin. Aesthet. Dermatol. 2017, 10, 34-44.

38. Sayed, K.S.; Hegazy, R.; Gawdat, H.I.; Abdel Hay, R.M.; Ahmed, M.M.; Mohammed, F.N.; Allam, R.; Fahim, A. The efficacy of intradermal injections of botulinum toxin in the management of enlarged facial pores and seborrhea: a split face-controlled study. J. Dermatol. Treat. 2020, 0, 1-7, doi:10.1080/09546634.2019.1708241.

39. Arredondo, J.; Hall, L.L.; Ndoye, A.; Nguyen, V.T.; Chernyavsky, A.I.; Bercovich, D.; Orr-Urtreger, A.; Beaudet, A.L.; Grando, S.A. Central role of fibroblast $\alpha 3$ nicotinic acetylcholine receptor in mediating cutaneous effects of nicotine. Lab. Invest. 2003, 83, 207-225, doi:10.1097/01.LAB.0000053917.46614.12.

40. Ahmed El Attar, Y.; Nofal, A. Microbotox for the treatment of wide facial pores: A promising therapeutic approach. J. Cosmet. Dermatol. 2020, 1-6, doi:10.1111/jocd.13675.

41. Diaspro, A.; Calvisi, L.; Manzoni, V.; Sito, G. Microbotulinum: A quantitative evaluation of aesthetic skin improvement in 62 patients. Plast. Reconstr. Surg. 2020, 987-994, doi:10.1097/PRS.0000000000007248.

42. Kapoor, R.; Shome, D.; Jain, V.; Dikshit, R. Facial rejuvenation after intradermal botulinum toxin: Is it really the botulinum toxin or is it the pricks? Dermatol. Surg. 2010, 36, 2098-2105, doi:10.1111/j.1524-4725.2010.01703.x.

43. Kondrateva, N.; Galkina, E.; Karakaeva, A.; Morrison, A.; Morrison, V. The effect of botulinum toxin type A injections on production of sebum in seborrhea. Saratov J. Med. Sci. Res. 2018, 14, 740-744.

44. Briganti, S.; Flori, E.; Mastrofrancesco, A.; Ottaviani, M. Acne as an altered dermato-endocrine response problem. Exp. Dermatol. 2020, 29, 833-839, doi:10.1111/exd.14168.

45. Plewig, G.; Melnik, B.; Chen, W. Plewig and Kligman's Acne and Rosacea; 4th ed.; Springer: Berlin, 2019.

46. Cunliffe, W.J. Acne; Martin Dunitz: London, 1989;

47. Ross, E.V. Optical treatments for acne. Dermatol. Ther. 2005, 18, 253-266, doi:10.1111/j.1529-8019.2005.05024.x.

48. Hana, A.; Booken, D.; Henrich, C.; Gratchev, A.; Maas-Szabowski, N.; Goerdt, S.; Kurzen, H. Functional significance of nonneuronal acetylcholine in skin epithelia. Life Sci. 2007, 80, 2214-2220, doi:10.1016/j.lfs.2007.02.007.

49. Klaz, I.; Kochba, I.; Shohat, T.; Zarka, S.; Brenner, S. Severe acne vulgaris and tobacco smoking in young men. J. Invest. Dermatol. 2006, 126, 1749-1752, doi:10.1038/sj.jid.5700326.

50. Capitanio, B.; Sinagra, J.L.; Ottaviani, M.; Bordignon, V.; Amantea, A.; Picardo, M. Acne and smoking. Dermatoendocrinol. 2009, 1, 129-135, doi:10.4161/derm.1.3.9638.

51. Lee, S.K. Multiple intradermal small bolus injection of botulinum toxin: The limit and the potentiality. J. Cosmet. Laser Ther. 2012, 14, 304-306, doi:10.3109/14764172.2012.738914.

52. Diamond, A.; Jankovic, J. Botulinum toxin in dermatology - Beyond wrinkles and sweat. J. Cosmet. Dermatol. $2006,5,169$.

53. Brin, M.F.; Boodhoo, T.I.; Pogoda, J.M.; James, L.M.; Demos, G.; Terashima, Y.; Gu, J.; Eadie, N.; Bowen, B.L. Safety and tolerability of onabotulinumtoxinA in the treatment of facial lines: A meta-analysis of individual patient data from global clinical registration studies in 1678 participants. J. Am. Acad. Dermatol. 2009, 61, 961-970.e11, doi:10.1016/j.jaad.2009.06.040.

54. Chiu, A.; Chon, S.Y.; Kimball, A.B. The response of skin disease to stress: Changes in the severity of acne vulgaris as affected by examination stress. Arch. Dermatol. 2003, 139, 4-7, doi:10.1001/archderm.139.7.897.

55. Zouboulis, C.C.; Böhm, M. Neuroendocrine regulation of sebocytes - A pathogenetic link between stress and acne. Exp. Dermatol., Suppl. 2004, 13, 31-35, doi:10.1111/j.1600-0625.2004.00254.x.

56. Orlowski, K.; Schnitger, A.; Glass, E.; Zouboulis, C.C. Corticotropin-releasing hormone skin signalling is receptor mediated and is predominant in the sebaceous glands. Exp. Dermatol. 2008, 13, 591-591, doi:10.1111/j.0906-6705.2004.00212.x. 
57. Mizuno, K.; Okuyama, K.; Akimoto, N.; Sato, T. Involvement of catecholamine in the differentiation of sebaceous glands in vitro. J. Invest. Dermatol. 2017, 137, s77, doi:https://doi.org/10.1016/j.jid.2017.02.467.

58. Borrel, V.; Thomas, P.; Catovic, C.; Racine, P.-J.; Konto-Ghiorghi, Y.; Lefeuvre, L.; Duclairoir-Poc, C.; Zouboulis, C.C.; Feuilloley, M.G.J. Acne and stress: Impact of catecholamines on Cutibacterium acnes. Front. Med. 2019, 6, doi:10.3389/fmed.2019.00155.

59. Zhou, Y.; Liu, Y.; Hao, Y.; Feng, Y.; Pan, L.; Liu, W.; Li, B.; Xiao, L.; Jin, L.; Nie, Z. The mechanism of botulinum A on Raynaud syndrome. Drug Des. Devel. Ther. 2018, 12, 1905-1915, doi:10.2147/DDDT.S161113.

60. Kim, H.J.; Seo, K.; Yum, K.W.; Oh, Y.S.; Yoon, T.G.; Yoon, S.M. Effects of botulinum toxin type A on the superior cervical ganglia in rabbits. Auton. Neurosci. Basic Clin. 2002, 102, 8-12, doi:10.1016/S1566-0702(02)00093-0.

61. Carroll, I.; Clark, J.D.; Mackey, S. Sympathetic block with botulinum toxin to treat complex regional pain syndrome. Ann. Neurol. 2009, 65, 348-351, doi:10.1002/ana.21601.

62. Lomneth, R.; Martin, T.F.J.; DasGupta, B.R. Botulinum neurotoxin light chain inhibits norepinephrine secretion in PC12 cells at an intracellular membranous or cytoskeletal site. J. Neurochem. 1991, 57, 1413-1421, doi:10.1111/j.1471-4159.1991.tb08308.x.

63. Banerjee, A.; Martin, T.F.J.; DasGupta, B.R. Nerve growth factor induces sensitivity to botulinum neurotoxin type A in norepinephrine-secreting PC12 cells. Neurosci. Lett. 1993, 164, 93-96, doi:10.1016/0304-3940(93)90865-I.

64. Morris, J.L.; Jobling, P.; Gibbins, I.L. Botulinum neurotoxin A attenuates release of norepinephrine but not NPY from vasoconstrictor neurons. Am. J. Physiol. - Hear. Circ. Physiol. 2002, 283, 2627-2635, doi:10.1152/ajpheart.00477.2002.

65. Toyoda, M.; Morohashi, M. New aspects in acne inflammation. Dermatology 2003, 206, 17-23, doi:10.1159/000067818.

66. Matak, I.; Tékus, V.; Bölcskei, K.; Lacković, Z.; Helyes, Z. Involvement of substance P in the antinociceptive effect of botulinum toxin type A: Evidence from knockout mice. Neuroscience 2017, 358, 137-145, doi:10.1016/j.neuroscience.2017.06.040.

67. Al-Ghamdi, A.S.; Alghanemy, N.; Joharji, H.; Al-Qahtani, D.; Alghamdi, H. Botulinum toxin: Non cosmetic and off-label dermatological uses. J. Dermatol. Dermatol. Surg. 2015, 19, 1-8, doi:10.1016/j.jdds.2014.06.002.

68. Cho, H.R.; Lew, B.L.; Lew, H.; Sim, W.Y. Treatment effects of intradermal botulinum toxin type A injection on alopecia areata. Dermatol. Surg. 2010, 36, 2175-2181, doi:10.1111/j.1524-4725.2010.01709.x.

69. Tamura, B.M.; Sortino-Rachou, A.M.; Cucé, L.C. Folliculitis responds to botulinum toxin: Is it possible? Dermatol. Surg. 2007, 33, 1398-1400, doi:10.1111/j.1524-4725.2007.33301.x.

70. Li, X.; He, C.; Chen, Z.; Zhou, C.; Gan, Y.; Jia, Y. A review of the role of sebum in the mechanism of acne pathogenesis. J. Cosmet. Dermatol. 2017, 16, 168-173, doi:10.1111/jocd.12345.

71. Alestas, T.; Ganceviciene, R.; Fimmel, S.; Müller-Decker, K.; Zouboulis, C.C. Enzymes involved in the biosynthesis of leukotriene B 4 and prostaglandin E 2 are active in sebaceous glands. J. Mol. Med. 2006, 84, 75-87, doi:10.1007/s00109-005-0715-8.

72. Ray, P.; Berman, J.D.; Middleton, W.; Brendle, J. Botulinum toxin inhibits arachidonic acid release associated with acetylcholine release from PC12 cells. J. Biol. Chem. 1993, 268, 11057-11064, doi:10.1016/s0021-9258(18)82091-9.

73. Chuang, Y.C.; Yoshimura, N.; Huang, C.C.; Wu, M.; Chiang, P.H.; Chancellor, M.B. Intraprostatic botulinum toxin A injection inhibits cyclooxygenase-2 expression and suppresses prostatic pain on capsaicin induced prostatitis model in rat. J. Urol. 2008, 180, 742-748, doi:10.1016/j.juro.2007.07.120.

74. Park, T.H. The effects of botulinum toxin A on mast cell activity: Preliminary results. Burns 2013, 39, 816-817, doi:10.1016/j.burns.2012.07.031.

75. Ramachandran, R.; Marino, M.J.; Paul, S.; Wang, Z.; Mascarenhas, N.L.; Pellett, S.; Johnson, E.A.; Dinardo, A.; Yaksh, T.L. A study and review of effects of botulinum toxins on mast cell dependent and independent pruritus. Toxins (Basel). 2018, 10, 113, doi:10.3390/toxins10040134.

76. Cao, L.F.; Si, M.; Huang, Y.; Chen, L.H.; Peng, X.Y.; Qin, Y.Q.; Liu, T.T.; Zhou, Y.; Liu, T.; Luo, W.F. Long-term anti-itch effect of botulinum neurotoxin A is associated with downregulation of TRPV1 and TRPA1 in the dorsal root ganglia in mice. Neuroreport 2017, 28, 518-526, doi:10.1097/WNR.0000000000000779.

77. Tóth, B.I.; Géczy, T.; Griger, Z.; Dózsa, A.; Seltmann, H.; Kovács, L.; Nagy, L.; Zouboulis, C.C.; Paus, R.; Bíró, T. Transient receptor potential vanilloid-1 signaling as a regulator of human sebocyte biology. J. Invest. Dermatol. 2009, 129, 329-339, doi:10.1038/jid.2008.258.

78. Makrantonaki, E.; Ganceviciene, R.; Zouboulis, C. An update on the role of the sebaceous gland in the pathogenesis of acne. Dermatoendocrinol. 2011, 3, 41-49, doi:10.4161/derm.3.1.13900. 